\title{
Excellent Image Quality or Superior Dose Conformity?
}

Joseph Stancanello ${ }^{1}$

1. GE Healthcare

$\square$ Corresponding author: Joseph Stancanello, joseph.stancanello@polimi.it

Disclosures can be found in Additional Information at the end of the article

\section{Abstract}

N/A

Categories: Radiation Oncology

Keywords: new devices, dose conformity, image quality

\section{Introduction And Background}

Recent trends in radiotherapy have focused primarily on 1) the use of imaging to visualize target and organs at risk (OAR) [1] and 2) on techniques able to deliver highly conformal treatments [2]: both aim to improve radiotherapy outcomes, for example, via dose escalation [3].

In the first group of developments, we reference the introduction of morphological, functional, and molecular imaging in all the steps of oncology workflow, from treatment planning to treatment delivery phases. Treatment plans based on multimodality images and daily imageguided radiotherapy (IGRT), based on volumetric images, are both becoming routine. Advanced examples of this trend are combinations of magnetic resonance imaging (MRI) [4] as well as positron emission tomography (PET) [5] scanners with treatment devices, such as linear accelerators or radioactive sources [6]. These new solutions claim the advantage of excellent imaging of the target and OARs using morphological/functional information prior or during treatment.

In the second group of developments, we could mention the widespread adoption of intensity modulated radiotherapy (IMRT) in all its variants and definitions, as well as of particle therapy developments with special focus on protons [7]. Motion management solutions and adaptive radiotherapy can be also regarded as ways to improve dose conformity [8]. Examples of this trend are HiART tomotherapy [9] and the CyberKnife [10] stereotaxy, both claiming an advantage of superior dose distributions.

One could argue if these trends are really two separate ways to allow safe and effective radiotherapy treatments - as appearances currently support - or if they are two sides of the same

\section{Published 07/20/2011}

\section{Copyright 2011}

Stancanello. This is an open access article distributed under the terms of the Creative Commons Attribution License CC-BY 3.0., which permits unrestricted use, distribution, and reproduction in any medium, provided the original author and source are credited. coin. In fact, on the basis of the ultimate goal of radiotherapy being maximizing tumor control probability while minimizing normal tissue complication probability, common ground could be clearly envisioned. The situation evokes the famous Menenius Agrippa apologue, where all the parts of the body cooperate to ensure survival of the whole. In such a manner, excellent image quality is a key enabler for accurately conformal treatments. Devices enabling excellent images suffer from dyspraxia if not coupled with the ability to deliver highly conformal treatments: why to accurately localize, in detail, the target and OARs if it is not possible to exploit this information to tailor highly conformal treatments? Conversely, devices enabling highly conformal treatments suffer from blindness if not coupled with excellent imaging systems: why have a device to tailor highly conformal treatments if the target and OARs cannot be accurately 
localized? In both cases, though, the benefits from image-guided and highly conformal treatments are per se extremely important; from a real-world view, they each seem to realize only partially the potential embedded in their combination.

Particular care is currently given to individualized treatments [11] where adaptive radiotherapy highlights inter- and intra-patient anatomical/functional differences. Early response monitoring images will play an increasing role so that the combination of MRI and PET scanners with radiotherapy devices will gain significant interest in the future.

\section{Review}

The emerging trend towards stereotactic radiosurgery (SRS) and stereotactic body radiotherapy (SBRT) requires the use of high soft tissue contrast images to minimize margins, as well as functional/molecular imaging to highlight the region to be selectively treated or boosted: smaller targets will open the way to higher doses per fraction. Dose conformity will allow nearby healthy tissue to be spared. There are some indications that many lesions could be treated in the future by SRS/SBRT, especially those benefiting from hypofractionated regimens once suitable protocols can be developed [12].

To give two examples, let us focus on emerging hypofractionated prostate [13] and breast [14] treatments. In this regimen, margins must be as small as possible, implying the need for the ability to distinguish moveable target interfaces and motion management solutions. In hypofractionated prostate treatments, the need for the identification of rectum-prostate-bladder interfaces requires high soft tissue contrast imaging, prior and during delivery. Then, highly conformal treatments with the help of adaptive radiotherapy and motion management accomplish the task of delivering safely hypofractionated treatments, limiting side-effects related to the high dose per fraction, such as rectum bleeding. In a similar way, accelerated partial breast irradiation, an emerging approach delivered via hypofractionated regimen, needs high soft tissue contrast imaging to distinguish the target from healthy breast tissue surrounding tumor bed. Like in prostate, highly conformal treatments focused on target limit side-effects, such as fibrosis: again, this is feasible only on the basis of what has been clearly identified.

Assuming that neither component should be compromised as the 'weak link' in complementary function, this leads to a theoretical conclusion that a hybrid device with the best morphological (or functional or molecular) imaging capabilities and highest dose conformity should represent the best tool for future developments in radiotherapy.

As a simplistic example, a compact proton accelerator integrated with MRI or PET imaging system - representing highest dose conformity and also "best" image quality - could become a focal point of research to make radiotherapy safer and more effective. It may be that the first step towards this development has already started, i.e., the use of in-room diagnostic-quality computer tomography (CT) in particle therapy facilities [15], and the idea to couple CT images to compact proton accelerator devices [16]. PET scanners make also it possible to visualize the dose delivered by proton therapy opening the way to in vivo dosimetry [17].

Another example is the combination of IMRT - in all its possible future evolutions - with phase contrast CT [18]. This x-ray technique holds the promise to distinguish soft tissue interfaces so that, for example, rectum-prostate-bladder interfaces could become well-identifiable in all cases. Due to the ring shape geometry of CT, rotational IMRT techniques would be a strong candidate to be coupled with phase contrast CT. New developments in detectors, tubes and reconstruction algorithms are likely to decrease the dose delivered for CT images to a level negligible compared to the overall therapeutic dose delivered during the course of the treatment. Thus, CT could become competitive to MRI, especially in therapy applications.

Extending the analysis also to other therapeutic approaches, another possible combination is 
high-intensity focused ultrasound (HIFU) [19] with MRI. The possibility of using MRI for anatomical and functional images as well as for MR-thermometry is likely to facilitate the exploitation of the untapped potential of HIFU with its non-ionizing nature, in all those cases where thermal ablation through ultrasound waves is feasible [20].

\section{Conclusions}

These examples are independent of cost and practicality. The large effort to develop some systems should be first somehow justified by the clinical gain it may bring, i.e. if the control or morbidity rates could be significantly improved not only from a theoretical but also from a practical point of view: is the game worth the candle? To answer this question, an evidencebased analysis is needed to understand if the theoretical advantages of these systems can be actually translated into reliable and repeatable improved outcomes in a large number of patients.

\section{Additional Information Disclosures}

This study did not involve human participants or tissue. This study did not involve animal subjects or tissue. Conflicts of interest: The authors have declared that no conflicts of interest exist except for the following: Financial relationships: Joseph Stancanello declare(s) an alternate financial activity from Siemens Healthcare. At that time I was an employee of Seimens Healthcare.

\section{References}

1. Chen GT, Sharp GC, Mori S: A review of image-guided radiotherapy. Radiol Phys Technol. 2009, 2:112. 10.1007/s12194-008-0045-y

2. Weber U, Kraft G: Comparison of carbon ions versus protons. Cancer J. 2009, 15:325-32. 10.1097/PPO.0b013e3181b01935

3. Biagioli MC, Hoffe SE: Emerging technologies in prostate cancer radiation therapy: improving the therapeutic window. Cancer Control. 2010, 17:223-32.

4. Lagendijk JJ, Raaymakers BW, Raaijmakers AJ, Overweg J, Brown KJ, Kerkhof EM, van der Put RW, Hårdemark B, van Vulpen M, van der Heide UA: MRI/linac integration. Radiother Oncol. 2008, 86:25-9. 10.1016/j.radonc.2007.10.034

5. Ishikawa M, Yamaguchi S, Tanabe S, Bengua G, Sutherland K, Suzuki R, Miyamoto N, Nishijima K, Katoh N, Shirato H: Conceptual design of PET-linac system for molecular-guided radiotherapy . Int J Radiat Oncol Biol Phys. 2010, 78:10.1016/j.ijrobp.2010.07.1566

6. http://www.viewray.com.

7. Mohan R, Bortfeld T: Proton therapy: Clinical gains through current and future treatment programs . Front Radiat Ther Oncol. 2011, 43:440-64. 10.1159/000322509

8. Ozhasoglu C, Saw CB, Chen H, Burton S, Komanduri K, Yue NJ, Huq SM, Heron DE: Synchrony-CyberKnife respiratory compensation technology. Med Dosim. 2008, 33:117-23. 10.1016/j.meddos.2008.02.004

9. Mackie TR, Balog J, Ruchala K, Shepard D, Aldridge S, Fitchard E, Reckwerdt P, Olivera G, McNutt T, Mehta M: Tomotherapy. Semin Radiat Oncol. 1999, 9:108-17.

10. Chang SD, Adler JR: Robotics and radiosurgery--The CyberKnife. Stereotact Funct Neurosurg. 2001, 76:204-8.

11. Stancanello J, Bayouth JE, Orton CG: Point/counterpoint. Genomics, functional and molecular imaging will pave the road to individualized radiation therapy. Med Phys. 2008, 35:4769-72.

12. Ko EC, Forsythe K, Buckstein M, Kao J, Rosenstein BS: Radiobiological rationale and clinical implications of hypofractionated radiation therapy. Cancer Radiother. 2011, 15:221-9. 10.1016/j.canrad.2010.12.007

13. King C: Stereotactic body radiotherapy for prostate cancer: Current results of a Phase II trial . Front Radiat Ther Oncol. 2011, 43:428-37. 10.1159/000322507

14. Beitsch PD, Shaitelman SF, Vicini FA: Accelerated partial breast irradiation. J Surg Oncol. 2011, 
103:362-8. 10.1002/jso.21785

15. Kress J, Minohara S, Endo M, Debus J, Kanai T: Patient position verification using CT images . Med Phys. 1999, 26:98-101.

16. Caporaso GJ, Mackie TR, Sampayan S, Chen YJ, Blackfield D, Harris J, Hawkins S, Holmes C, Nelson S, Paul A, Poole B, Rhodes M, Sanders D, Sullivan J, Wang L, Watson J, Reckwerdt PJ, Schmidt R, Pearson D, Flynn RW, Matthews D, Purdy J: A compact linac for intensity-modulated proton therapy based on a dielectric wall accelerator. Phys Med. 2008, 24:98-101. 10.1016/j.ejmp.2008.01.010

17. Knopf A, Parodi K, Paganetti H, Cascio E, Bonab A, Bortfeld T: Quantitative assessment of the physical potential of proton beam range verification with PET/CT. Phys Med Biol. 2008, 53:4137-51. 10.1088/0031-9155/53/15/009

18. Raupach R, Flohr TG: Analytical evaluation of the signal and noise propagation in x-ray differential phase-contrast computed tomography. Phys Med Biol. 2011, 56:2219-44. 10.1088/00319155/56/7/020

19. Zhou YF: High intensity focused ultrasound in clinical tumor ablation . World J Clin Oncol. 2011, 2:8-27. 10.5306/wjco.v2.i1.8

20. Benedict S, De Meerleer G, Orton CG, Stancanello J: High intensity focused ultrasound may be superior to radiation therapy for the treatment of early stage prostate cancer. Med Phys. 2011, 38:409-11. 